\title{
Selection Dynamics of Carcinogenesis
}

\author{
Bala Srinivasan ${ }^{1}$, Udayabaskaran Swaminathan ${ }^{2} \&$ Zahir Hussain Buhari ${ }^{3}$ \\ ${ }^{1}$ Department of Statistics, Madras Christian College (Autonomous), Tambaram, \\ Chennai 600059, Tamil Nadu, India. \\ ${ }^{2}$ Department of Mathematics, Vel Tech Dr. RR \& Dr. SR Technical University, Avadi, Chennai 600062, India. \\ ${ }^{3}$ Department of General Studies, Yanbu Industrial College, P.O. Box 30436, Yanbu Al Sinaiyah, Yanbu, \\ Kingdom of Saudi Arabia.
}

\begin{abstract}
In this paper, we analyze a stochastic model of tumorigenesis which takes into account the selection dynamics in a heterogeneous colony of cells. The stochastic model is a modified branching process which includes cell divisions, death and mutation. Mutation is included explicitly to account for two types of intermediate cells. The model is essentially a Markov branching process evolving in a random environment. Using the regeneration technique, we obtain integral equations for the probability generating functions of the underlying stochastic variables.
\end{abstract}

\section{Introduction}

Branching processes have been used by several researchers for studying biological processes (see Harris [7], Athereya and Ney [4] and Assmussen and Herin [3]. Typical biological processes such as carcinogenesis exhibit selection and mutation in their evolution. A great amount of theoretical investigations on carcinogenesis have been made during the past several decades (see for example, Armitage and Doll [2], Kendall [8] Waugh [15], Knudson [9], Moolgavkar and Venzon [12], Moolgavkar and Luebeck [11], Michor et al. [10] and Beerenwinkel and Sullivant [5]). Several monographs have appeared containing almost all research on theoretical aspects of carcinogenesis (see for example, Tan [13], Frank [6] and Weinberg [16]). Recently, Thalhauser et al. [14] have studied the selection dynamics in a heterogeneous spatial colony of cells by using spatial generalization of birth-and-death processes which include migration. They analyzed the impact of migration on the ability of a single mutant cell to invade a pre-existing colony. Environmental factors such as ionizing radiation, chemical pollution, smoking and alcohol consumption have been proved to initiate tumorigenesis of various organs (see Actis and Eynard [1]). Furthermore the bipolar chromosome segregation errors are caused by the transient spindle defects observed in cancer cells. This aspect has not been given due importance in the previous stochastic models which consider selection and mutation in their evolution. In the present paper, we fill this gap by studying a branching process model of selection and mutation in their evolution subject to a random environment. Accordingly, the object of the present paper is to propose and analyze a stochastic model of a cell division process taking into account the effect of environmental influences on the selection of life-time distribution.

The organization of the chapter is as follows:

In section 2, a branching process model is described. Section 3 provides the derivation of explicit expressions for the time-dependent mean size of the population.

In section 4, a numerical illustration is provided to exhibit the model.

\section{Description Of The Model}

We consider a stochastic population of cells which evolves in a carcinogenic environment. The cells are of three types, namely normal cells, intermediate cells and malignant cells. Due to the impact of the environment, the intermediate cells are of two subtypes. Let $\mathrm{X}(\mathrm{t})$ be the number of normal cells in the population at time t. Let $Z_{11}(t)$ and $Z_{12}(t)$ denote the number of intermediate stage cells of type 1 and type 2, respectively. Let $\mathrm{W}(\mathrm{t})$ be the number of malignant cells at time $\mathrm{t}$. We assume that in a small interval of time $(\mathrm{t}, \mathrm{t}+$ $\Delta)$

1. a normal cell divides into two normal cells independent of the environment with probability $\mathrm{L} \Delta+\mathrm{o}(\Delta)$;

2. a normal cell divides into one normal cell and one intermediate cell of type 1 under the influence of the environment with probability $\mathrm{Np} \Delta+\mathrm{o}(\Delta)$;

3. a normal cell divides into one normal cell and one intermediate cell of type 2 under the influence of the environment with probability $\mathrm{N}(1-\mathrm{p}) \Delta+\mathrm{o}(\Delta)$;

4. a normal cell dies or differentiates with probability $\mathrm{D} \Delta+\mathrm{o}(\Delta)$;

5. an intermediate cell of type 1 divides into two intermediate cells of type 1 with probability $\alpha_{11} \Delta+\mathrm{o}(\Delta)$; 
6. an intermediate cell of type 1 divides into one intermediate cells of type 1 and one intermediate cell of type 2 under the influence of the environment with probability $\alpha_{12} \Delta+\mathrm{o}(\Delta)$;

7. an intermediate cell of type 2 divides into two intermediate cells of type 2 independent of the environment with probability $\alpha_{22} \Delta+\mathrm{o}(\Delta)$;

8. an intermediate cell of type 2 divides into one intermediate cells of type 2 and one intermediate cell of type 1 under the influence of the environment with probability $\alpha_{21} \Delta+\mathrm{o}(\Delta)$;

9. an intermediate cell of type 1 divides into one intermediate cell of type 1 and one malignant cell under the influence of the environment with probability $\beta_{1} \Delta+\mathrm{o}(\Delta)$;

10. an intermediate cell of type 2 divides into one intermediate cell of type 2 and one malignant cell under the influence of the environment with probability $\beta_{2} \Delta+\mathrm{o}(\Delta)$;

11. an intermediate cell of any type dies or differentiates independent of the environment with probability $\mu \Delta+o(\Delta)$.

We consider the vector random variable $\xi(t)=\left(X(t), Z_{1}(t), Z_{2}(t), W(t)\right)$ and define

$$
\psi\left(x, z_{1}, z_{2}, w, t\right)=\sum_{i, m \cdot n, k=0}^{\infty} P\left\{\xi(t)=(i, m, n, k \mid \xi(0)=(1,0,0,0)\} x^{j} z_{1}^{m} z_{2}{ }^{n} w^{k} .\right.
$$

By the expectation operator $\mathrm{E}$, we get

$$
\psi\left(x, z_{1}, z_{2}, w, t\right)=E\left[x^{X(t)} z_{1}^{Z_{1}(t)} z_{2}^{Z_{2}(t)} w^{W(t)} \mid X(0)=1, Z_{1}(0)=0, Z_{2}(0)=0, W(0)=0\right]
$$

which denotes the probability generating function for the number of normal cells, type 1 intermediate cells, type 2 intermediate cells, and malignant cells at time t, starting with one normal cell, no intermediate cells and malignant cells at time 0 . Next we consider the random vectors $\xi(t)=\left(Z_{1}(t), Z_{2}(t), W(t)\right)$ and define

$$
\begin{aligned}
& \phi_{1}\left(z_{1}, z_{2}, w, t\right)=\sum_{n=0}^{\infty} \sum_{w=0}^{\infty} P\left\{\zeta_{j}(t)=\left(z_{j}, k\right) \mid \zeta(0)=(1,0,0)\right\} z_{j}{ }^{n} w^{k}, \\
& \phi_{2}\left(z_{1}, z_{2}, w, t\right)=\sum_{n=0}^{\infty} \sum_{w=0}^{\infty} P\left\{\zeta_{j}(t)=\left(z_{j}, k\right) \mid \zeta(0)=(0,1,0)\right\} z_{j}{ }^{n} w^{k} .
\end{aligned}
$$

By the expectation operator $\mathrm{E}$, we get

$$
\begin{aligned}
& \phi_{1}\left(z_{1}, z_{2}, w, t\right)=E\left[z_{1}{ }^{Z_{1}(t)} z_{2}{ }^{Z_{2}(t)} w^{W(t)} \mid Z_{1}(0)=1, Z_{2}(0)=0, W(0)=0\right], \\
& \phi_{2}\left(z_{1}, z_{2}, w, t\right)=E\left[z_{1}{ }^{Z_{1}(t)} z_{2}{ }^{Z_{2}(t)} w^{W(t)} \mid Z_{1}(0)=0, Z_{2}(0)=1, W(0)=0\right] .
\end{aligned}
$$

$\phi_{j}\left(z_{1}, z_{2}, w, t\right)$ represents the probability generating function for the number of intermediate cells of types 1 and 2 and malignant cells at time $t$, starting with one intermediate cell of type $\mathrm{j}$ and no malignant cell at time 0. Using the regeneration method of Kendall [8], we derive integral equations for the probability generating functions $\psi\left(x, z_{1}, z_{2}, w, t\right), \phi_{1}\left(z_{1}, z_{2}, w, t\right)$ and $\phi_{2}\left(z_{1}, z_{2}, w, t\right)$. To achieve this, we first note that either no event occurs in the interval $(0, t)$ or at least one event occurs in $(0, t)$.Using the total probability law, we get

$$
\begin{aligned}
& \psi(t)=x e^{-K t}+\int_{0}^{t} e^{-K u}\left[L \psi^{2}(t-u)+N p \psi(t-u) \phi_{1}(t-u)\right. \\
& \left.+N(1-p) \psi(t-u) \phi_{2}(t-u)+D\right] d u, \\
& \phi_{1}(t)=z_{1} e^{-k_{1}(t)}+\int_{0}^{t} e^{-k_{1} u}\left[\alpha_{11} \phi_{1}^{2}(t-u)+\alpha_{12} \phi_{1}(t-u) \phi_{2}(t-u)+\beta_{1} \phi_{1}(t-u) w+\mu\right] d u, \\
& \phi_{2}(t)=z_{2} e^{-k_{2}(t)}+\int_{0}^{t} e^{-k_{2} u}\left[\alpha_{22} \phi_{2}^{2}(t-u)+\alpha_{21} \phi_{1}(t-u) \phi_{2}(t-u)+\beta_{2} \phi_{2}(t-u) w+\mu\right] d u,
\end{aligned}
$$

where $\mathrm{K}=\mathrm{L}+\mathrm{N}+\mathrm{D}, k_{1}=\alpha_{11}+\alpha_{12}+\beta_{1}+\mu$ and $k_{2}=\alpha_{22}+\alpha_{21}+\beta_{2}+\mu$.By a simple technique, equations (2.1), (2.2) and (2.3) can be equivalently written as differential equations:

$$
\frac{d \psi(t)}{d t}+K \psi(t)=L \psi^{2}(t)+N p \psi(t) \phi_{1}(t)+N(1-p) \psi(t) \phi_{2}(t)+D
$$


$\frac{d \phi_{1}(t)}{d t}+k_{1} \phi_{1}(t)=\alpha_{11} \phi_{1}^{2}(t)+\alpha_{12} \phi_{1}(t) \phi_{2}(t)+\beta_{1} \phi_{1}(t) w+\mu$,

$\frac{d \phi_{2}(t)}{d t}+k_{2} \phi_{1}(t)=\alpha_{22} \phi_{2}{ }^{2}(t)+\alpha_{21} \phi_{1}(t) \phi_{2}(t)+\beta_{2} \phi_{2}(t) w+\mu$.

Equations (2.4)-(2.6) are subject to the initial conditions

$\psi(x, z, w, 0)=x, \phi_{1}\left(z_{1}, z_{2}, w, 0\right)=z_{1}, \phi_{2}\left(z_{1}, z_{2}, w, 0\right)=z_{2}$.

\section{Mean Number Of Cells}

We define the conditional means

$$
\begin{aligned}
& M_{X}(t)=\varepsilon\left\{X(t) \mid X(0)=1, Z_{1}(0)=0, Z_{2}(0)=0, W(0)=0\right\}, \\
& M_{Z_{1}}(t)=\varepsilon\left\{Z_{1}(t) \mid X(0)=1, Z_{1}(0)=0, Z_{2}(0)=0, W(0)=0\right\}, \\
& M_{Z_{2}}(t)=\varepsilon\left\{Z_{2}(t) \mid X(0)=1, Z_{1}(0)=0, Z_{2}(0)=0, W(0)=0\right\}, \\
& m_{11}(t)=\varepsilon\left\{Z_{1}(t) \mid Z_{1}(0)=1, Z_{2}(0)=0, W(0)=0\right\}, \\
& m_{12}(t)=\varepsilon\left\{Z_{2}(t) \mid Z_{1}(0)=1, Z_{2}(0)=0, W(0)=0\right\}, \\
& m_{21}(t)=\varepsilon\left\{Z_{1}(t) \mid Z_{1}(0)=0, Z_{2}(0)=1, W(0)=0\right\}, \\
& m_{22}(t)=\varepsilon\left\{Z_{2}(t) \mid Z_{1}(0)=0, Z_{2}(0)=1, W(0)=0\right\} .
\end{aligned}
$$

Using (2.1)-(2.3), we obtain

$$
\begin{aligned}
& M_{X}(t)=e^{-K t}+\int_{0}^{t} e^{-K u}(2 L+N) M_{X}(t-u) d u, \\
& M_{Z_{1}}(t)=\int_{0}^{t} e^{-K u}\left[(2 L+N) M_{Z_{1}}(t-u)+N p m_{11}(t-u)+N(1-p) m_{21}(t-u)\right] d u, \\
& M_{Z_{2}}(t)=\int_{0}^{t} e^{-K u}\left[(2 L+N) M_{Z_{2}}(t-u)+N p m_{12}(t-u)+N(1-p) m_{22}(t-u)\right] d u, \\
& m_{11}(t)=e^{-k_{1}(t)}+\int_{0}^{t} e^{-k_{1} u}\left[\left\{2 \alpha_{11}+\alpha_{12}+\beta_{1}\right\} m_{11}(t-u)+\alpha_{12} m_{21}(t-u)\right] d u, \\
& m_{12}(t)=\int_{0}^{t} e^{-k_{1} u}\left[\left\{2 \alpha_{11}+\alpha_{12}+\beta_{1}\right\} m_{12}(t-u)+\alpha_{12} m_{22}(t-u)\right] d u, \\
& m_{21}(t)=\int_{0}^{t} e^{-k_{2} u}\left[\left\{2 \alpha_{22}+\alpha_{21}+\beta_{2}\right\} m_{21}(t-u)+\alpha_{21} m_{11}(t-u)\right] d u, \\
& m_{22}(t)=e^{-k_{2} t}+\int_{0}^{t} e^{-k_{2} u}\left[\left\{2 \alpha_{22}+\alpha_{21}+\beta_{2}\right\} m_{11}(t-u)+\alpha_{21} m_{12}(t-u)\right] d u .
\end{aligned}
$$

Using Laplace transform, equations (3.1)-(3.7) give

$$
\begin{aligned}
& (s+D-L) M_{X}{ }^{*}(s)=1, \\
& (s+D-L) M_{Z_{1}}{ }^{*}(s)=N p m_{11}{ }^{*}(s)+N(1-p) p m_{21}{ }^{*}(s), \\
& (s+D-L) M_{Z_{2}}{ }^{*}(s)=N p m_{12}{ }^{*}(s)+N(1-p) p m_{22}{ }^{*}(s), \\
& \left(s+\mu-\alpha_{11}\right) m_{11}{ }^{*}(s)=1+\alpha_{12} m_{21}{ }^{*}(s), \\
& \left(s+\mu-\alpha_{11}\right) m_{12}{ }^{*}(s)=\alpha_{12} m_{22}{ }^{*}(s), \\
& \left(s+\mu-\alpha_{22}\right) m_{21}{ }^{*}(s)=\alpha_{21} m_{11}{ }^{*}(s),
\end{aligned}
$$


$\left(s+\mu-\alpha_{22}\right) m_{22}{ }^{*}(s)=1+\alpha_{21} m_{12}{ }^{*}(s)$,

where we used the Laplace transform notation

$f^{*}(s)=\int_{0}^{\infty} e^{-s t} f(t) d t$.

Solving (3.8)-(3.14), we get

$m_{11}^{*}(s)=\frac{s+\mu-\alpha_{22}}{s\left(s+2 \mu-\alpha_{11}-\alpha_{22}\right)}$,

$m_{12}^{*}(s)=\frac{\alpha_{12}}{s\left(s+2 \mu-\alpha_{11}-\alpha_{22}\right)}$,

$m_{21}{ }^{*}(s)=\frac{\alpha_{21}}{s\left(s+2 \mu-\alpha_{11}-\alpha_{22}\right)}$,

$m_{22}{ }^{*}(s)=\frac{s+\mu-\alpha_{11}}{s\left(s+2 \mu-\alpha_{11}-\alpha_{22}\right)}$,

Equations (3.1)-(3.7) are exactly solvable and we obtain

$$
\begin{aligned}
& M_{X}(t)=e^{(L-D) t}, \\
& m_{11}(t)=\frac{1}{2 \mu-\alpha_{11}-\alpha_{22}}\left[\left(\mu-\alpha_{22}\right)+\left(\mu-\alpha_{11}\right) e^{\left(\alpha_{11}+\alpha_{22}-\mu\right) t}\right], \\
& m_{12}(t)=\frac{1}{2 \mu-\alpha_{11}-\alpha_{22}}\left[\alpha_{12}-\alpha_{12} e^{\left(\alpha_{11}+\alpha_{22}-\mu\right) t}\right], \\
& m_{21}(t)=\frac{1}{2 \mu-\alpha_{11}-\alpha_{22}}\left[\alpha_{21}-\alpha_{21} e^{\left(\alpha_{11}+\alpha_{22}-\mu\right) t}\right], \\
& m_{22}(t)=\frac{1}{2 \mu-\alpha_{11}-\alpha_{22}}\left[\left(\mu-\alpha_{11}\right)+\left(\mu-\alpha_{22}\right) e^{\left(\alpha_{11}+\alpha_{22}-\mu\right) t}\right], \\
& \left.M_{Z_{1}}(t)=N e^{(L-D) t} \subseteq\left[p m_{11}(t)\right)+(1-p) m_{21}(t)\right], \\
& \left.M_{Z_{2}}(t)=N e^{(L-D) t} \subseteq\left[p m_{12}(t)\right)+(1-p) m_{22}(t)\right],
\end{aligned}
$$

where (C) symbol is used in the following sense:

$f(t) @ g(t)=\int_{0}^{t} f(u) g(t-u) d u$.

\section{Numerical Illustration}

In this section, we illustrate our model by adopting the same values for the values of the parameters as in Moolgavkar and Venzon [12]:

$\mathrm{D}=\mu=0$

$L=\alpha_{11}=\alpha_{12}=\alpha_{21}=\alpha_{22}=0.7$;

$p=0.5$

$N=\beta_{1}=\beta_{2}=1 \times 10^{-6}$.

We compute the mean number of intermediate cells from time 0 to time 12 and plot $M_{Z_{1}}(t)$ against t.Thegraph is depicted in Fig. 1. It shows that the mean number increases with respect to time. 


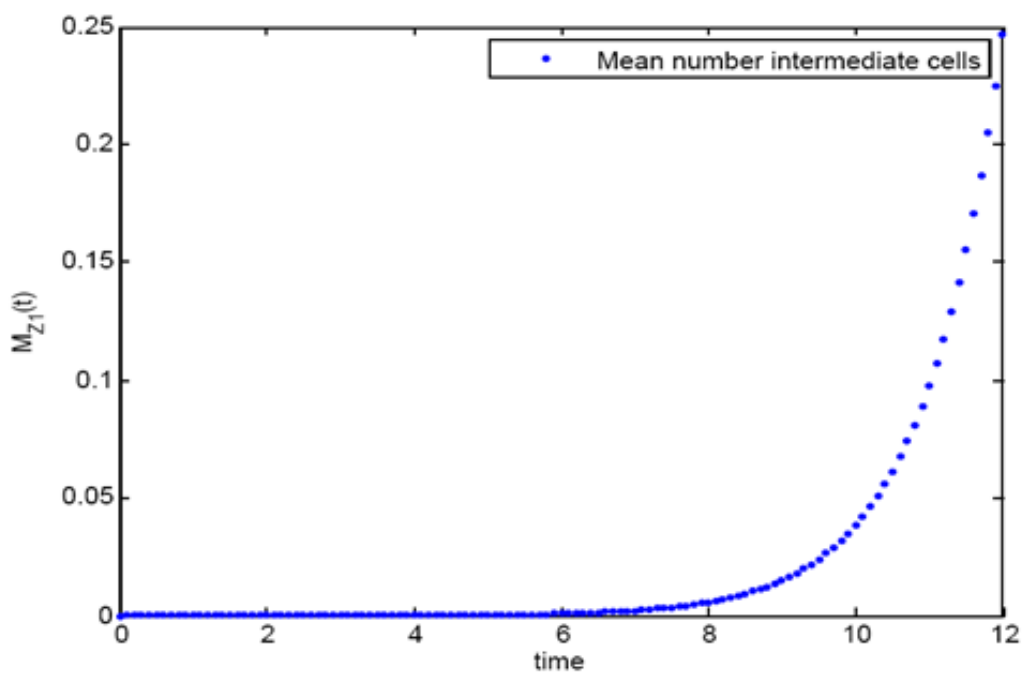

Figure 1

\section{Bibliography}

[1]. Actis, A. B., and Eynard, A. R., 2000. “'Influence of environmental and nutritional factors on salivary gland tumorigenesis with a special reference to dietary lipids," European Journal of Clinical Nutrition, Vol. 54, pp. 805-810.

[2]. Armitage, P. and Doll, R., 1957. "A two-stage theory of carcinogenesis in relation to the age distribution of human cancer," British Jour. of Cancer, Vol. 11, pp. 161-169.

[3]. Asmussen, S. and Hering, H., 1983. "Branching Processes," Birkhauser, Boston.

[4]. Athreya, K. B. and Ney, P. E., 1972. “Branching Processes," Berlin: Springer.

[5]. Beerenwinkel, N. and Sullivant, S., 2008. Markov models for accumulating mutations, J. Theor. Biol. 242, 409-420.

[6]. Frank, S., "'Dynamics of Cancer Incidence, Inheritance and Evolution," Oxford: Princeton University Press, 2007.

[7]. Harris, T. E., 1963. “The Theory of Branching Processes," Berlin: Springer

[8]. Kendal, D. G., 1960. “'Birth-and-Death processes, and the theory of carcinogenesis, Biometrika 47, 13-22.

[9]. Knudson, A. G., 1971. “Mutation and cancer: statistical study of retinoblastoma," Proc. Nat. Acad. Sci. USA., Vol. 68, pp. 820823.

[10]. Michor, F., Iwasa, Y., and Nowak, M. A., 2004. “'Dynamics of cancer progression," Nat. Rev. Can. 4, 197-205.

[11]. Moolgavkar, S. H. and Luebeck, E. G., 1992. J. natl. Cancer Inst. 84, 610-618.

[12]. Moolgavkar, S. H. and Venzon, D. J., 1979. “Two event model for carcinogenesis: Incidence curves for childhood and adult tumors," Math. Biosci., Vol. 47, pp. 55-77.

[13]. Tan, W. Y., “'Stochastic Models of Carcinogenesis," Marcel Dekker, New York, 1991

[14]. Thalhauser, C. J., Lowengrub, J. S., Stupack, D., and Komarova, N. L., 2010. "'Selection in spatial stochastic models of cancer: Migration as a key modulator of fitness," Biology Direct, 5:21.

[15]. Waugh, W. A. O'N., 1961. “Age-dependence in a stochastic model of carcinogenesis," Proceedings of the Fourth Berkeley Symposium on Mathematical Statistics and Probability, Berkeley and Los Angeles, University of California Press, Vol. 4, 405-413.

[16]. Weinberg, R. A., “The Biology of Cancer", Garland Science (Taylor and Francis Group), 2007. 